\title{
ON IDEALS GENERATED BY FOLD PRODUCTS OF LINEAR FORMS
}

\author{
ŞTEFAN O. TOHǍNEANU
}

\begin{abstract}
Let $\mathbb{K}$ be a field of characteristic 0 . Given $n$ linear forms in $R=\mathbb{K}\left[x_{1}, \ldots, x_{k}\right]$, with no two proportional, in one of our main results we show that the ideal $I \subset R$ generated by all $(n-2)$-fold products of these linear forms has linear graded free resolution. This result helps determining a complete set of generators of the symmetric ideal of $I$. Via Sylvester forms we can analyze from a different perspective the generators of the presentation ideal of the Orlik-Terao algebra of the second order; this is the algebra generated by the reciprocals of the products of any two (distinct) of the linear forms considered. We also show that when $k=2$, and when the collection of $n$ linear forms may contain proportional linear forms, for any $1 \leq a \leq n$, the ideal generated by $a$-fold products of these linear forms has linear graded free resolution.
\end{abstract}

\section{INTRODUCTION}

Let $R:=\mathbb{K}\left[x_{1}, \ldots, x_{k}\right]$ be the ring of (homogeneous) polynomials with coefficients in $\mathbb{K}$, a field of characteristic 0 , with the natural grading. Denote $\mathfrak{m}:=\left\langle x_{1}, \ldots, x_{k}\right\rangle$ to be the irrelevant maximal ideal of $R$. Let $\ell_{1}, \ldots, \ell_{n}$ be linear forms in $R$, some possibly proportional, and denote this collection by $\Sigma=$ $\left(\ell_{1}, \ldots, \ell_{n}\right) \subset R$; we need a notation to take into account the fact that some of these linear forms are proportional. For $\ell \in \Sigma$, by $\Sigma \backslash\{\ell\}$ we will understand the collection of linear forms of $\Sigma$ from which $\ell$ has been removed. Also, we denote $|\Sigma|=n$.

Let $1 \leq a \leq n$ be an integer and define the ideal generated by a-fold products of $\Sigma$ to be the ideal of $R$

$$
I_{a}(\Sigma):=\left\langle\left\{\ell_{i_{1}} \cdots \ell_{i_{a}} \mid 1 \leq i_{1}<\cdots<i_{a} \leq n\right\}\right\rangle .
$$

We also make the convention $I_{0}(\Sigma):=R$, and $I_{b}(\Sigma)=0$, for all $b>n$. Also, if $\Sigma=\emptyset, I_{a}(\Sigma)=0$, for any $a \geq 1$.

A homogeneous ideal $I \subset R$ generated in degree $d$ it is said to have linear graded free resolution, if one has the graded free resolution

$$
0 \rightarrow R^{n_{b+1}}(-(d+b)) \rightarrow \cdots \rightarrow R^{n_{2}}(-(d+1)) \rightarrow R^{n_{1}}(-d) \rightarrow R \rightarrow R / I \rightarrow 0,
$$

for some positive integer $b$. By convention, the zero ideal has linear graded free resolution.

Though only recently has been written down (see [1, Conjecture 1]), it has been five years at least since it has been conjectured that for any $\Sigma \subset R$, and any $1 \leq a \leq|\Sigma|$, the ideals $I_{a}(\Sigma)$ have linear graded free resolution.

Without any loss of generality, we can assume that $\langle\Sigma\rangle=\mathfrak{m}$; otherwise, after a change of variables we can assume that $\Sigma \subset \mathbb{K}\left[x_{1}, \ldots, x_{s}\right], s<k$, with $\langle\Sigma\rangle=\left\langle x_{1}, \ldots, x_{s}\right\rangle$. Suppose $|\Sigma|=n$. Here are some instances when it is known that this conjecture is true:

(1) Let $\mathcal{C}_{\Sigma}$ be the linear code with generating matrix having columns dual to the linear forms of $\Sigma$ (in no particular order). This will be a linear code of length $n$, and dimension $k$. Suppose its minimum (Hamming) distance is $d$. Then, by [18, Theorem 3.1], for any $1 \leq a \leq d$, we have $I_{a}(\Sigma)=\mathfrak{m}^{a}$, and in these ideals have linear graded free resolution (see for example [8, Corollary 1.5]).

2010 Mathematics Subject Classification. Primary 13D02; Secondary 52C35, 14N20, 13A30.

Key words and phrases. linear free resolution, ideals generated by products of linear forms, hyperplane arrangements, OrlikTerao algebra, special fiber, symmetric ideal.

Department of Mathematics, University of Idaho, Moscow, Idaho 83844-1103, USA, Email: tohaneanu@uidaho.edu, Phone: 208-885-6234, Fax: 208-885-5843. 
(2) With the same point of view from (1), for some $\Sigma$ with certain properties, in [2, Theorem 3.1], it is shown that $I_{d+1}(\Sigma)$ has linear graded free resolution (of course, after using [8]).

(3) If any $k$ of the linear forms in $\Sigma$ are linearly independent (i.e., the linear code $\mathcal{C}_{\Sigma}$ is Maximum Distance Separable code), then $I_{a}(\Sigma)$ has linear graded free resolution, for any $1 \leq a \leq n$. To see this, apply the proof of [9, Theorem 2.5] and [1, Proposition 3.5].

(4) More generally than part (3), whenever $R / I_{a}(\Sigma)$ is Cohen-Macaulay, then $I_{a}(\Sigma)$ has a linear graded free resolution. This can be seen from the discussions at the end of the proof of [19, Proposition 2.1], and immediately after it; the point there is that the Eagon-Northcott complex becomes the desired linear graded free resolution of $R / I_{a}(\Sigma)$ (see [6, Theorem A2.60]).

(5) If no two of the linear forms of $\Sigma$ are proportional, then from part (4), $I_{n-1}(\Sigma)$, so $a=n-1$, has linear graded free resolution.

(6) If $\langle\Sigma\rangle=\langle\ell\rangle$, for some $\ell \in R_{1}$, then for any $1 \leq a \leq n$, we have $I_{a}(\Sigma)=\left\langle\ell^{a}\right\rangle$, which, as any principal ideal, has linear graded free resolution.

(7) $I_{n}(\Sigma)$, so $a=n$, is the principal ideal generated by $\prod_{\ell \in \Sigma} \ell$, hence it has linear graded free resolution.

In this article we add to the list above three more nontrivial cases when the conjecture is true:

- For any $k \geq 1$ and for any $\Sigma \subset R=\mathbb{K}\left[x_{1}, \ldots, x_{k}\right]$, a collection of $n$ linear forms with no two proportional, $I_{n-2}(\Sigma)$, so $a=n-2$, has linear graded free resolution. (see Theorem 2.4)

- If $k=2$, then for any $\Sigma \subset \mathbb{K}\left[x_{1}, x_{2}\right]_{1}$, and for any $1 \leq a \leq|\Sigma|, I_{a}(\Sigma)$ has linear graded free resolution. (see Theorem 2.2)

- Generalizing part (5) above, for any $\Sigma \subset R=\mathbb{K}\left[x_{1}, \ldots, x_{k}\right], I_{n-1}(\Sigma)$ has linear graded free resolution. (see Section 2.1)

As applications to the main result Theorem 2.4, we find a criterion when $R / I_{n-2}(\Sigma)$ is Cohen-Macaulay (Corollary 2.5), and when $k=3$, we determine a primary decomposition of $I_{n-2}(\Sigma)$ (Proposition 2.6).

Let $\Sigma=\left(\ell_{1}, \ldots, \ell_{n}\right) \subset R=\mathbb{K}\left[x_{1}, \ldots, x_{k}\right]$ be a collection of linear forms such that $\operatorname{gcd}\left(\ell_{i}, \ell_{j}\right)=1$, if $i \neq j$. Let $\mathcal{A}$ be the central hyperplane arrangement defined by $\ell_{1}, \ldots, \ell_{n}$, i.e., $\mathcal{A}=\left\{V\left(\ell_{1}\right), \ldots, V\left(\ell_{n}\right)\right\} \subset$ $\mathbb{P}^{k-1}$. In such instance, instead of writing $\Sigma=\left(\ell_{1}, \ldots, \ell_{n}\right) \subset R$, we will write $\mathcal{A}=\left\{\ell_{1}, \ldots, \ell_{n}\right\} \subset R$. The $\operatorname{rank}$ of $\mathcal{A}$ is $\operatorname{rank}(\mathcal{A})=\operatorname{ht}\left(\left\langle\ell_{1}, \ldots, \ell_{n}\right\rangle\right)$; if $\operatorname{rank}(\mathcal{A})=k$, then $\mathcal{A}$ is called essential.

In recent years, especially after the work of [17] and [14], there has been a lot of focus on the algebra $O T(\mathcal{A}):=\mathbb{K}\left[\frac{1}{\ell_{1}}, \ldots, \frac{1}{\ell_{n}}\right]$, called the Orlik-Terao algebra (after the names of the mathematicians who first introduced it in [13]), or the algebra of the reciprocal plane. Even more recently, in the hyperplane arrangements community discussions have started in regard to studying $\mathbb{K}\left[\ldots, \frac{1}{\prod_{i \in I} \ell_{i}}, \ldots\right]$, where $I$ runs over all independent sets of $\mathcal{A}$, of certain given size. Due to [10, Theorem 2.4], the study of these new algebras can be done by analyzing the special fiber of certain ideals of $R$, generated by products of linear forms. Since for any $1 \leq i<j \leq n$, the set $\{i, j\}$ is independent, this is the path we are pursuing for some parts of Section 3 , where we analyse some of the first properties of the algebra

$$
O T(2, \mathcal{A}):=\mathbb{K}\left[\ldots, \frac{1}{\ell_{i} \ell_{j}}, \ldots\right], 1 \leq i<j \leq n,
$$

that we are calling the Orlik-Terao algebra of second order of $\mathcal{A}$.

Propositions 3.3 and 3.4 show that there is a strong connection between $O T(2, \mathcal{A})$ and $O T(\mathcal{A})$, yet despite that the generators of $I(\mathcal{A}) \subset \mathbb{K}\left[y_{1}, \ldots, y_{n}\right]$, the presentation ideal of $O T(\mathcal{A})$, have nice combinatorial description (they are "boundaries" of circuits, see [13]), it is not the same for the generators of $I(2, \mathcal{A}) \subset$ $\mathbb{K}\left[\ldots, t_{i, j}, \ldots\right]$, the presentation ideal of $O T(2, \mathcal{A})$. The difficulties occur due to the fact that the elements of $I(2, \mathcal{A})$ are obtained from the elements of $I(\mathcal{A}) \cap \mathbb{K}\left[\ldots, y_{i} y_{j}, \ldots\right]$, and though in theory this looks reasonable, in practice the task to find them is challenging (see, e.g., Example 3.5). By [10, Theorem 2.4], $O T(2, \mathcal{A})$ is isomorphic to the special fiber of the ideal $I_{n-2}(\mathcal{A}) \subset R$, so by following the approach of [10, 
Proposition 3.5], one can obtain elements in $I(2, \mathcal{A})$ from the generators of the symmetric ideal of $I_{n-2}(\mathcal{A})$, via Sylvester forms. But even with this technique, as it is well known in the Ress algebras / elimination theory community, no one guarantees that one obtains all the generators of $I(2, \mathcal{A})$. We conjecture that we do obtain all of them. None-the-less, our main result (Theorem 2.4 helps determine all the generators of the symmetric ideal of $I_{n-2}(\mathcal{A})$ (see Proposition 3.6).

\section{IDEALS WITH LINEAR FREE RESOLUTION}

Let $\Sigma=\left(\ell_{1}, \ldots, \ell_{n}\right)$ be a collection of linear forms in $R:=\mathbb{K}\left[x_{1}, \ldots, x_{k}\right]$, some of them, possibly proportional. Let $1 \leq a \leq n$ be an integer and consider the ideal of $R$

$$
I_{a}(\Sigma):=\left\langle\left\{\ell_{i_{1}} \cdots \ell_{i_{a}} \mid 1 \leq i_{1}<\cdots<i_{a} \leq n\right\}\right\rangle .
$$

In this section we show that for the cases listed in the Introduction, $I_{a}(\Sigma)$ has linear graded free resolution. And we also look at some consequences of these results.

2.1. The case $a=n-1$. Suppose $\Sigma=(\underbrace{\ell_{1}, \ldots, \ell_{1}}_{n_{1}}, \ldots, \underbrace{\ell_{s}, \ldots, \ell_{s}}_{n_{s}})$, with $n_{1}, \ldots, n_{s} \geq 1$, and $\operatorname{gcd}\left(\ell_{i}, \ell_{j}\right)=$

1 , for any $1 \leq i<j \leq s$. Suppose $|\Sigma|=n_{1}+\cdots+n_{s}=n$.

Let $\Sigma_{0}=\left\{\ell_{1}, \ldots, \ell_{s}\right\}$. It is immediate to observe that

$$
I_{n-1}(\Sigma)=\left(\ell_{1}^{n_{1}-1} \cdots \ell_{s}^{n_{s}-1}\right) I_{s-1}\left(\Sigma_{0}\right) .
$$

By the proof of [10, Lemma 3.1(a)], we have the graded free resolution

$$
0 \rightarrow R(-s)^{s-1} \rightarrow R(-(s-1))^{s} \rightarrow R \rightarrow R / I_{s-1}\left(\Sigma_{0}\right) \rightarrow 0 .
$$

By Hilbert-Burch Theorem ([7, Theorem 20.15]), we obtain the linear graded free resolution

$$
0 \rightarrow R(-n)^{s-1} \rightarrow R(-(n-1))^{s} \rightarrow R \rightarrow R / I_{n-1}(\Sigma) \rightarrow 0 .
$$

2.2. The case $k=2$. Suppose $\Sigma=\left(\ell_{1}, \ldots, \ell_{n}\right) \subset R=\mathbb{K}[x, y]$. Suppose that the linear form $\ell$ shows up at least twice in $\Sigma$. Let $\Sigma^{\prime}:=\Sigma \backslash\{\ell\}$, and $\Sigma^{\prime \prime}:=\Sigma^{\prime} \backslash\{\ell\}$.

Lemma 2.1. Let $1 \leq a \leq n$. If

(i) $I_{a-1}\left(\Sigma^{\prime}\right)$ has linear graded free resolution, and

(ii) $I_{a}\left(\Sigma^{\prime}\right): \ell=I_{a-1}\left(\Sigma^{\prime \prime}\right)$,

then $I_{a}(\Sigma): \ell=I_{a-1}\left(\Sigma^{\prime}\right)$ and $I_{a}(\Sigma)$ has linear graded free resolution.

Proof. First we show that $I_{a}(\Sigma): \ell=I_{a-1}\left(\Sigma^{\prime}\right)$.

Since we have $I_{a}(\Sigma)=\ell I_{a-1}\left(\Sigma^{\prime}\right)+I_{a}\left(\Sigma^{\prime}\right)$, the inclusion " $\supseteq$ " is clear.

Let $f \in I_{a}(\Sigma): \ell$. Then $\ell f=\ell g+h$, for some $g \in I_{a-1}\left(\Sigma^{\prime}\right)$ and $h \in I_{a}\left(\Sigma^{\prime}\right)$. Therefore, $\ell(f-g) \in$ $I_{a}\left(\Sigma^{\prime}\right)$, leading to $f-g \in I_{a}\left(\Sigma^{\prime}\right): \ell$. From condition (ii), we get $f-g \in I_{a-1}\left(\Sigma^{\prime \prime}\right)$. But $\Sigma^{\prime \prime} \subset \Sigma^{\prime}$, so $f \in I_{a-1}\left(\Sigma^{\prime}\right)$. This gives the other inclusion.

Without any loss of generality, suppose $\ell=x$. Then for each $i=1, \ldots, n, \ell_{i}=b_{i} x+c_{i} y$, where $b_{i}, c_{i} \in \mathbb{K}$ some of them equal to zero. Suppose that precisely $1 \leq s \leq n-2$ of the $c_{i}$ 's are NOT equal to zero; if $s=0$, then $\Sigma=(\underbrace{x, \ldots, x}_{n})$, and part (6) in the Introduction shows that $I_{a}(\Sigma)$ has linear graded free resolution.

Let $\bar{\Sigma}:=(\underbrace{y, \ldots, y}_{s})$. Then

$$
\left\langle I_{a}(\Sigma), x\right\rangle=\left\langle I_{a}(\bar{\Sigma}), x\right\rangle .
$$

If $a>s$, by convention, $I_{a}(\bar{\Sigma})=0$.

Everything put together gives the short exact sequence of $R$-modules 


$$
0 \rightarrow R(-1) / I_{a-1}\left(\Sigma^{\prime}\right) \stackrel{\cdot x}{\rightarrow} R / I_{a}(\Sigma) \rightarrow R /\left\langle I_{a}(\bar{\Sigma}), x\right\rangle \rightarrow 0 .
$$

Condition (i), together with [8, Theorem 1.2 (2)], gives the Castelnuovo-Mumford regularity $\operatorname{reg}_{R}\left(R(-1) / I_{a-1}\left(\Sigma^{\prime}\right)\right)=a-2+1=a-1$.

If $a>s$, then $\operatorname{reg}_{R}\left(R /\left\langle I_{a}(\bar{\Sigma}), x\right\rangle\right)=0$, and if $a \leq s$, then $\operatorname{reg}_{R}\left(R /\left\langle I_{a}(\bar{\Sigma}), x\right\rangle\right)=a-1$.

But, from the inequalities of regularities under a short exact sequence (see [6, Corollary $20.19 \mathrm{~b}$.$] )$

$$
\operatorname{reg}\left(R / I_{a}(\Sigma)\right) \leq \max \left\{\operatorname{reg}_{R}\left(R(-1) / I_{a-1}\left(\Sigma^{\prime}\right)\right), \operatorname{reg}_{R}\left(R /\left\langle I_{a}(\bar{\Sigma}), x\right\rangle\right)\right\} \leq a-1 .
$$

Since $I_{a}(\Sigma)$ is generated in degree $a$, we get that $\operatorname{reg}\left(R / I_{a}(\Sigma)\right)=a-1$, and hence, from [8, Theorem $1.2(2)], I_{a}(\Sigma)$ has linear graded free resolution.

Theorem 2.2. Let $\Sigma=\left(\ell_{1}, \ldots, \ell_{n}\right) \subset R=\mathbb{K}[x, y]$, be a collection of linear forms, some possibly proportional. Then, for any $1 \leq a \leq n, I_{a}(\Sigma)$ has linear graded free resolution.

Proof. Let $\mathcal{A}$ be the reduced support of $\Sigma$; i.e., $\mathcal{A}$ consists of all nonproportional elements of $\Sigma$. If we show that $I_{b}(\mathcal{A})$ has linear graded free resolution, and $I_{b}(\mathcal{A}): \ell=I_{b-1}(\mathcal{A} \backslash\{\ell\})$, for any $\ell \in \mathcal{A}$, and any $1 \leq b \leq|\mathcal{A}|$, then via Lemma 2.1 and the conventions in the Introduction, by adding one-by-one linear forms according to their multiplicity to obtain $\Sigma$, we obtain that $I_{a}(\Sigma)$ has linear graded resolution as well, for any $1 \leq a \leq n$.

Suppose $\mathcal{A}=\left\{\ell_{1}, \ldots, \ell_{m}\right\}, m \leq n$, and $\operatorname{gcd}\left(\ell_{i}, \ell_{j}\right)=1$, for all $1 \leq i<j \leq m$. If $m=1$, then part (6) of the introduction shows directly that $I_{a}(\Sigma)$ has linear graded free resolution.

Suppose $m \geq 2$. Then, $\operatorname{rank}(\mathcal{A})=2$. Let $\mathcal{C}_{\mathcal{A}}$ be the linear code with generating matrix $G$ having columns dual to the linear forms of $\mathcal{A}$. This code has length $m$ and dimension 2 . Since any two of the linear forms of $\mathcal{A}$ are linearly independent, the maximum number of columns of $G$ that span a $2-1=1$ dimensional vector space is 1 . So the minimum distance of $\mathcal{C}_{\mathcal{A}}$ is $m-1$ (see, e.g., [20, Remark 2.2]). But in these condition, by [18, Theorem 3.1], we have indeed that for any $1 \leq b \leq m-1$,

$$
I_{b}(\mathcal{A})=\langle x, y\rangle^{b} \text {. }
$$

Also $I_{m}(\mathcal{A})=\left\langle\ell_{1} \cdots \ell_{m}\right\rangle$.

It is clear that for any $1 \leq b \leq m, I_{b}(\mathcal{A})$ has linear graded free resolution, and that $I_{b}(\mathcal{A}): \ell=$ $I_{b-1}(\mathcal{A} \backslash\{\ell\})$, for any $\ell \in \mathcal{A}$.

2.3. The case $a=n-2$. Let $\mathcal{A}=\left\{\ell_{1}, \ldots, \ell_{n}\right\} \subset R=\mathbb{K}\left[x_{1}, \ldots, x_{k}\right]$ be a hyperplane arrangement. Our main goal in this section is to show that the graded $R$-module $R / I_{n-2}(\mathcal{A})$ has linear graded free resolution. For $1 \leq i<j \leq n$, denote

$$
f_{i, j}:=\frac{\ell_{1} \ell_{2} \cdots \ell_{n}}{\ell_{i} \ell_{j}} \in R
$$

the generators of the ideal $I_{n-2}(\mathcal{A})$.

Consider the complex of (graded) $R$-modules:

$$
\mathbf{C C}(\mathcal{A}): 0 \rightarrow \bigoplus_{1 \leq i<j \leq n} \frac{R(-(n-2))}{\left\langle\ell_{i}, \ell_{j}\right\rangle} \stackrel{\phi_{\mathcal{A}}}{\rightarrow} \frac{R}{I_{n-1}(\mathcal{A})} \stackrel{\pi_{\mathcal{A}}}{\rightarrow} \frac{R}{I_{n-2}(\mathcal{A})} \rightarrow 0
$$

where the map $\pi_{\mathcal{A}}$ is the natural surjection defined from the inclusion $I_{n-1}(\mathcal{A}) \subset I_{n-2}(\mathcal{A})$ and the map $\phi_{\mathcal{A}}$ is defined as

$$
\phi_{\mathcal{A}}\left(\ldots, \widehat{h}_{i, j}, \ldots\right)=\left[\sum_{1 \leq i<j \leq n} h_{i, j}\left(\prod_{u \in[n] \backslash\{i, j\}} \ell_{u}\right)\right] \bmod I_{n-1}(\mathcal{A}),
$$

where $\widehat{h}_{i, j} \in \frac{R}{\left\langle\ell_{i}, \ell_{j}\right\rangle}$.

Obviously, $\prod_{u \in[n] \backslash\{i, j\}} \ell_{u} \in I_{n-2}(\mathcal{A})$. This gives us that 
- $\phi_{\mathcal{A}}$ is well-defined: if $h_{i, j}, g_{i, j} \in R$ are such that $h_{i, j}-g_{i, j} \in\left\langle\ell_{i}, \ell_{j}\right\rangle$, then obviously

$$
\left(h_{i, j}-g_{i, j}\right)\left(\prod_{u \in[n] \backslash\{i, j\}} \ell_{j}\right) \in I_{n-1}(\mathcal{A}) .
$$

- $\operatorname{Im}\left(\phi_{\mathcal{A}}\right)=\frac{I_{n-2}(\mathcal{A})}{I_{n-1}(\mathcal{A})}=\operatorname{ker}\left(\pi_{\mathcal{A}}\right)$.

A 3-dependency is a linear combination among exactly three of the linear forms of $\mathcal{A}$. Suppose

$$
\mathrm{D}_{i_{1}, i_{2}, i_{3}}: c_{i_{1}} \ell_{i_{1}}+c_{i_{2}} \ell_{i_{2}}+c_{i_{3}} \ell_{i_{3}}=0,1 \leq i_{1}<i_{2}<i_{3} \leq n,
$$

is such a 3-dependency, where $c_{i_{1}}, c_{i_{2}}, c_{i_{3}} \in \mathbb{K} \backslash\{0\}$. In matroid language we say that $\left\{i_{1}, i_{2}, i_{3}\right\}$ is a circuit.

Let $F_{i_{1}, i_{2}, i_{3}}:=\frac{\ell_{1} \ell_{2} \cdots \ell_{n}}{\ell_{i_{1}} \ell_{i_{2}} \ell_{i_{3}}} \in R$. Then, by multiplying the dependency $\mathrm{D}_{i_{1}, i_{2}, i_{3}}$ with $F_{i_{1}, i_{2}, i_{3}}$, one obtains

$$
c_{i_{1}} f_{i_{2}, i_{3}}+c_{i_{2}} f_{i_{1}, i_{3}}+c_{i_{3}} f_{i_{1}, i_{2}}=0 .
$$

By denoting with $\Lambda(\mathcal{A})$ to be the left-most $R$-module in the complex above, i.e.

we just obtained that the element of $\Lambda(\mathcal{A})$

$$
\Lambda(\mathcal{A}):=\bigoplus_{1 \leq i<j \leq n} \frac{R}{\left\langle\ell_{i}, \ell_{j}\right\rangle}
$$

$$
\widehat{\mathbf{c}}_{i_{1}, i_{2}, i_{3}}:=(0, \ldots, 0, \underbrace{\widehat{c}_{i_{3}}}_{\left(i_{1}, i_{2}\right)}, 0, \ldots, 0, \underbrace{\widehat{c}_{i_{2}}}_{\left(i_{1}, i_{3}\right)}, 0, \ldots, 0, \underbrace{\widehat{c}_{i_{1}}}_{\left(i_{2}, i_{3}\right)}, 0, \ldots, 0)
$$

is an element of the kernel of $\phi_{\mathcal{A}}$; the "underbraces" specify the position (or the summand) in the module $\Lambda(\mathcal{A})$. In fact we have that the entire cyclic $R$-submodule $\mathcal{R}_{i_{1}, i_{2}, i_{3}}:=R \cdot \widehat{\mathbf{c}}_{i_{1}, i_{2}, i_{3}}$ is included in $\operatorname{ker}\left(\phi_{\mathcal{A}}\right)$, leading to

$$
\Lambda_{3}(\mathcal{A}) \subseteq \operatorname{ker}\left(\phi_{\mathcal{A}}\right)
$$

where $\Lambda_{3}(\mathcal{A})$ denotes the (internal) direct sum of the $R$-submodules $\mathcal{R}_{i_{1}, i_{2}, i_{3}}$ of $\Lambda(\mathcal{A})$, running over all circuits $\left\{i_{1}, i_{2}, i_{3}\right\}$ of $\mathcal{A}$.

Lemma 2.3. We have $\Lambda_{3}(\mathcal{A})=\operatorname{ker}\left(\phi_{\mathcal{A}}\right)$.

We are going to prove the lemma a bit later.

Let $\mathcal{V}(\mathcal{A}):=\Lambda_{3}(\mathcal{A}) \otimes_{R} \mathbb{K}$ be the $\mathbb{K}$-vector subspace of $\mathbb{K}^{m}$, where $m:=\left(\begin{array}{l}n \\ 2\end{array}\right)$. Let $p(\mathcal{A}):=\operatorname{dim}_{\mathbb{K}} \mathcal{V}(\mathcal{A})$, be the number of "independent" 3-dependencies of $\mathcal{A}$. In Claim 1 in the subsection following the proof of the main result, we prove that $p(\mathcal{A})=\sum_{X \in L_{2}(\mathcal{A})}\left(\begin{array}{c}\left|\mathcal{A}_{X}\right|-1 \\ 2\end{array}\right)$.

With these notations, our first main result of this paper is the following.

Theorem 2.4. Let $\mathcal{A}=\left\{\ell_{1}, \ldots, \ell_{n}\right\} \subset R$ be a hyperplane arrangement. Then the $R$-module $R / I_{n-2}(\mathcal{A})$ has graded linear free resolution

$0 \rightarrow R^{m-n-p(\mathcal{A})+1}(-n) \rightarrow R^{2 m-n-2 p(\mathcal{A})}(-(n-1)) \rightarrow R^{m-p(\mathcal{A})}(-(n-2)) \rightarrow R \rightarrow R / I_{n-2}(\mathcal{A}) \rightarrow 0$.

Proof. From Lemma 2.3 we have the acyclic (graded) complex of $R$-modules:

$$
0 \rightarrow \Lambda_{3}(\mathcal{A})(-(n-2)) \rightarrow \Lambda(\mathcal{A})(-(n-2)) \stackrel{\phi_{\mathcal{A}}}{\longrightarrow} \frac{R}{I_{n-1}(\mathcal{A})} \stackrel{\pi_{\mathcal{A}}}{\rightarrow} \frac{R}{I_{n-2}(\mathcal{A})} \rightarrow 0 .
$$

Since each $R /\left\langle\ell_{i}, \ell_{j}\right\rangle, i \neq j$ is isomorphic as $R$-modules with $T:=\mathbb{K}\left[x_{1}, \ldots, x_{k-2}\right]$, if $k \geq 3$, or $T:=\mathbb{K}$, if $k=2$, then we have the isomorphism of $R$-modules

$$
\Lambda_{3}(\mathcal{A}) \simeq T^{p(\mathcal{A})} .
$$


Since $\left\langle\ell_{i}, \ell_{j}\right\rangle, i \neq j$ is a complete intersection, the $R$-module $T \simeq R /\left\langle\ell_{i}, \ell_{j}\right\rangle$ has the (Koszul) linear free resolution

$$
0 \rightarrow R(-2) \rightarrow R^{2}(-1) \rightarrow R \rightarrow T \rightarrow 0
$$

This leads to the linear free resolutions:

$$
0 \rightarrow R^{p(\mathcal{A})}(-n) \rightarrow R^{2 p(\mathcal{A})}(-(n-1)) \rightarrow R^{p(\mathcal{A})}(-(n-2)) \rightarrow \Lambda_{3}(\mathcal{A})(-(n-2)) \rightarrow 0,
$$

and

$$
0 \rightarrow R^{m}(-n) \rightarrow R^{2 m}(-(n-1)) \rightarrow R^{m}(-(n-2)) \rightarrow \Lambda(\mathcal{A})(-(n-2)) \rightarrow 0 .
$$

Then, via mapping cone, the (graded) $R$-module $\operatorname{Im}\left(\phi_{\mathcal{A}}\right)$, has a free resolution:

$$
0 \rightarrow R^{p(\mathcal{A})}(-n) \rightarrow \begin{gathered}
R^{2 p(\mathcal{A})}(-(n-1)) \\
R^{m}(-n)
\end{gathered} \rightarrow \begin{gathered}
R^{p(\mathcal{A})}(-(n-2)) \\
R^{2 m}(-(n-1))
\end{gathered} \rightarrow R^{m}(-(n-2)) \rightarrow \operatorname{Im}\left(\phi_{\mathcal{A}}\right) \rightarrow 0 .
$$

By [8, Lemma 1.13], $\operatorname{Im}\left(\phi_{\mathcal{A}}\right)$ has linear free resolution, and this can be obtained from the above resolution via appropriate "cancelations":

$$
0 \rightarrow R^{m-p(\mathcal{A})}(-n) \rightarrow R^{2 m-2 p(\mathcal{A})}(-(n-1)) \rightarrow R^{m-p(\mathcal{A})}(-(n-2)) \rightarrow \operatorname{Im}\left(\phi_{\mathcal{A}}\right) \rightarrow 0 .
$$

The $R$-module, $R / I_{n-1}(\mathcal{A})$ has linear free resolution (see, e.g., [15, Lemma 3.2])

$$
0 \rightarrow R^{n-1}(-n) \rightarrow R^{n}(-(n-1)) \rightarrow R \rightarrow R / I_{n-1}(\mathcal{A}) \rightarrow 0 .
$$

Then again by mapping cone, since $\operatorname{Im}\left(\phi_{\mathcal{A}}\right)=\operatorname{ker}\left(\pi_{\mathcal{A}}\right)$, we obtain the free resolution

$$
0 \rightarrow R^{m-p(\mathcal{A})}(-n) \rightarrow \begin{gathered}
R^{2 m-2 p(\mathcal{A})}(-(n-1)) \\
R^{n-1}(-n)
\end{gathered} \rightarrow \begin{gathered}
R^{m-p(\mathcal{A})}(-(n-2)) \\
R^{n}(-(n-1))
\end{gathered} \rightarrow R \rightarrow R / I_{n-2}(\mathcal{A}) \rightarrow 0 .
$$

Using again [8, Lemma 1.13], $R / I_{n-2}(\mathcal{A})$ has a linear free resolution, and after the appropriate cancellations the linear free resolution is the one claimed in the statement.

2.3.1. Proof of Lemma 2.3 Suppose $X_{1}, \ldots, X_{e}$ are all the rank 2 flats in $L(\mathcal{A})$, the lattice of intersections of $\mathcal{A}$, with $n_{u}:=\left|\mathcal{A}_{X_{u}}\right| \geq 3$ for all $u=1, \ldots, e$. For a flat $X \in L(\mathcal{A}), \mathcal{A}_{X}$ denotes the subset of hyperplanes of $\mathcal{A}$ that contain $X$.

- Claim 1: One has

$$
\Lambda_{3}(\mathcal{A}) \cong \bigoplus_{u=1}^{e} \Lambda_{3}\left(\mathcal{A}_{X_{u}}\right) \text { and } p(\mathcal{A})=\operatorname{dim}_{\mathbb{K}} \mathcal{V}(\mathcal{A})=\sum_{u=1}^{e}\left(\begin{array}{c}
n_{u}-1 \\
2
\end{array}\right)
$$

Proof Claim 1. If $\left\{i_{1}, i_{2}, i_{3}\right\}$ is a circuit, then $X:=V\left(\ell_{i_{1}}, \ell_{i_{2}}, \ell_{i_{3}}\right)$ is a rank 2 flat with $\left|\mathcal{A}_{X}\right| \geq 3$. If a non-zero tuple belongs to $\Lambda_{3}\left(\mathcal{A}_{X_{u}}\right) \cap \Lambda_{3}\left(\mathcal{A}_{X_{v}}\right), u \neq v$, then it has at least one non-zero entry, say the $(i, j)$-th entry, that gives that there exist a circuit $\{i, j, a\}$ of $\mathcal{A}_{X_{u}}$, and a circuit $\{i, j, b\}$ of $\mathcal{A}_{X_{v}}$. Since $X_{u}=V\left(\ell_{i}, \ell_{j}\right)$ and $X_{v}=V\left(\ell_{i}, \ell_{j}\right)$, we get a contradiction.

For the second part we have to show that if $X$ is a rank 2 flat with $s:=\left|\mathcal{A}_{X}\right| \geq 3$, then $p\left(\mathcal{A}_{X}\right)=$ $\left(\begin{array}{c}s-1 \\ 2\end{array}\right)$. After a change of variables and some reordering of the hyperplanes in $\mathcal{A}$, we can suppose $\mathcal{A}_{X}=$ $\left\{\ell_{1}, \ldots, \ell_{s}\right\} \subset \mathbb{K}\left[x_{1}, x_{2}\right] \subset R$, with $\ell_{1}=x_{1}$, and $\ell_{i}=x_{1}+\lambda_{i} x_{2}, \lambda_{i} \in \mathbb{K} \backslash\{0\}, i=2, \ldots, s$, and $\lambda_{i} \neq \lambda_{j}$, if $i \neq j$.

Any three of the linear forms of $\mathcal{A}_{X}$ lead to a dependency, and for any $2 \leq u<v<w \leq s$ we have 


$$
\begin{aligned}
\mathrm{D}_{u, v, w}: & \left(\lambda_{v}-\lambda_{w}\right) \ell_{u}+\left(\lambda_{w}-\lambda_{u}\right) \ell_{v}+\left(\lambda_{u}-\lambda_{v}\right) \ell_{w}=0 \\
\mathrm{D}_{1, u, v}: & \left(\lambda_{u}-\lambda_{v}\right) \ell_{1}+\lambda_{v} \ell_{u}+\left(-\lambda_{u}\right) \ell_{v}=0 \\
\mathrm{D}_{1, u, w}: & \left(\lambda_{u}-\lambda_{w}\right) \ell_{1}+\lambda_{w} \ell_{u}+\left(-\lambda_{u}\right) \ell_{w}=0 .
\end{aligned}
$$

It is easy to check that

which leads to

$$
\mathrm{D}_{u, v, w}=\frac{1}{\lambda_{u}}\left[\left(\lambda_{u}-\lambda_{w}\right) \mathrm{D}_{1, u, v}-\left(\lambda_{u}-\lambda_{v}\right) \mathrm{D}_{1, u, w}\right]
$$

$$
\mathbf{c}_{u, v, w}=\frac{1}{\lambda_{u}}\left[\left(\lambda_{u}-\lambda_{w}\right) \mathbf{c}_{1, u, v}-\left(\lambda_{u}-\lambda_{v}\right) \mathbf{c}_{1, u, w}\right] .
$$

This means that $\mathcal{V}\left(\mathcal{A}_{X}\right)$ is generated by the vectors $\mathbf{c}_{1, u, v}$, where $2 \leq u<v \leq s$.

Suppose there exist $\gamma_{u, v} \in \mathbb{K}, 2 \leq u<v \leq s$, such that $\mathbf{c}:=\sum_{2 \leq u<v \leq s} \gamma_{u, v} \mathbf{c}_{1, u, v}=0$. For each pair $2 \leq u<v \leq s$, the $(u, v)$-entry of $\mathbf{c}$ equals to $\gamma_{u, v}\left(\lambda_{u}-\lambda_{v}\right)$, which must be zero. Since $\lambda_{u} \neq \lambda_{v}$, we get that $\gamma_{u, v}=0$.

So $\left\{\mathbf{c}_{1, u, v} \mid 2 \leq u<v \leq s\right\}$ is a basis for $\mathcal{V}\left(\mathcal{A}_{X}\right)$, and hence the claim.

- Claim 2: Lemma 2.3 is true if $n=2$.

Proof Claim 2. We have $\mathcal{A}=\left\{\ell_{1}, \ell_{2}\right\} \subset R, \operatorname{gcd}\left(\ell_{1}, \ell_{2}\right)=1$. Obviously, $\Lambda_{3}(\mathcal{A})=0$. With the convention that $I_{0}(\mathcal{A})=R$, and since $I_{1}(\mathcal{A})=\left\langle\ell_{1}, \ell_{2}\right\rangle$, then the complex $\mathbf{C C}(\mathcal{A})$ translates into the complex

$$
0 \rightarrow \frac{R}{\left\langle\ell_{1}, \ell_{2}\right\rangle} \stackrel{\phi_{\mathcal{A}}}{\longrightarrow} \frac{R}{\left\langle\ell_{1}, \ell_{2}\right\rangle} \rightarrow 0
$$

where $\phi_{\mathcal{A}}$ is the identity map, and hence $\operatorname{ker}\left(\phi_{\mathcal{A}}\right)$ is also zero.

- Claim 3: Lemma2.3 is true if $k=2$.

Proof Claim 3. Let $\mathcal{A}=\left\{\ell_{1}, \ldots, \ell_{n}\right\} \subset R:=\mathbb{K}\left[x_{1}, x_{2}\right]$ with $\operatorname{gcd}\left(\ell_{i}, \ell_{j}\right)=1$, if $i \neq j$. From Claim 2, suppose $n \geq 3$. Let us look at the map $\phi_{\mathcal{A}}$ :

with

$$
\bigoplus_{1 \leq i<j \leq n} \frac{R(-(n-2))}{\left\langle\ell_{i}, \ell_{j}\right\rangle} \stackrel{\phi_{\mathcal{A}}}{\longrightarrow} \frac{R}{I_{n-1}(\mathcal{A})}
$$

$$
\phi_{\mathcal{A}}\left(\ldots, \widehat{h}_{i, j}, \ldots\right)=\left[\sum_{1 \leq i<j \leq n} h_{i, j}\left(\prod_{u \in[n] \backslash\{i, j\}} \ell_{u}\right)\right] \bmod I_{n-1}(\mathcal{A}) .
$$

Since for any $i \neq j,\left\langle\ell_{i}, \ell_{j}\right\rangle=\mathfrak{m}=\left\langle x_{1}, x_{2}\right\rangle$, if degree of $h_{i, j}$ is $\geq 1$, then $\widehat{h}_{i, j}=0$ in $R /\left\langle\ell_{i}, \ell_{j}\right\rangle$.

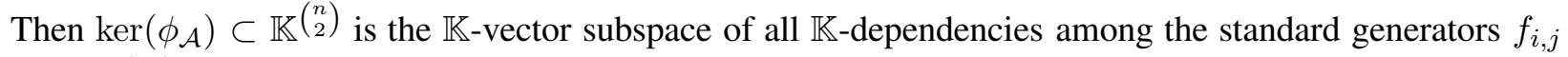
of $I_{n-2}(\mathcal{A})$. So

$$
\operatorname{dim}_{\mathbb{K}} \operatorname{ker}\left(\phi_{\mathcal{A}}\right)=\left(\begin{array}{l}
n \\
2
\end{array}\right)-\mu\left(I_{n-2}(\mathcal{A})\right)
$$

where $\mu\left(I_{n-2}(\mathcal{A})\right)$ is the minimum number of generators of $I_{n-2}(\mathcal{A})$.

Same coding theory argument as in the proof of Theorem 2.2 gives

$$
I_{n-2}(\mathcal{A})=\left\langle x_{1}, x_{2}\right\rangle^{n-2} .
$$

Therefore, $\mu\left(I_{n-2}(\mathcal{A})\right)=n-1$, leading to

$$
\operatorname{dim}_{\mathbb{K}} \operatorname{ker}\left(\phi_{\mathcal{A}}\right)=\left(\begin{array}{l}
n \\
2
\end{array}\right)-(n-1)=\left(\begin{array}{c}
n-1 \\
2
\end{array}\right)
$$

But from the proof of the second part of Claim 1, this is exactly $p(\mathcal{A})$, leading to $\Lambda_{3}(\mathcal{A})=\operatorname{ker}\left(\phi_{\mathcal{A}}\right)$. 
At this moment we proceed to prove Lemma 2.3, Let $\mathcal{A}=\left\{\ell_{1}, \ldots, \ell_{n}\right\} \subset R:=\mathbb{K}\left[x_{1}, \ldots, x_{k}\right], k \geq 2$ with $\operatorname{gcd}\left(\ell_{i}, \ell_{j}\right)=1$, if $i \neq j$.

We will use induction on $|\mathcal{A}|=n \geq 2$. From Claim 2, the base case $n=2$ is verified.

Suppose $n \geq 3$. For $1 \leq i<j \leq n$, let $h_{i, j} \in R$, such that $\phi_{\mathcal{A}}\left(\ldots, \widehat{h}_{i, j}, \ldots\right)=0$ in $R / I_{n-1}(\mathcal{A})$. So

$$
\mathbf{P}:=\sum_{1 \leq i<j \leq n} h_{i, j}\left(\prod_{u \in[n] \backslash\{i, j\}} \ell_{u}\right) \in I_{n-1}(\mathcal{A}) .
$$
3.

If $\operatorname{rank}(\mathcal{A})=2$, then modulo a change of variables, Claim 3 proves the result. So assume that $\operatorname{rank}(\mathcal{A}) \geq$

For any $1 \leq i<j \leq n$, there exists $u(i, j) \in[n] \backslash\{i, j\}$ such that

$$
\ell_{u(i, j)} \notin\left\langle\ell_{i}, \ell_{j}\right\rangle \text {. }
$$

This is true because, otherwise there would exist $1 \leq i_{0}<j_{0} \leq n$ such that for all $v=1, \ldots, n$, we would have $\ell_{v} \in\left\langle\ell_{i_{0}}, \ell_{j_{0}}\right\rangle$, and $\operatorname{so} \operatorname{rank}(\mathcal{A})=2$; a contradiction with the assumption we made above.

Without any loss of generality, suppose $h_{1,2} \neq 0$, and suppose $u(1,2)=n$. Let $\mathcal{A}^{\prime}:=\mathcal{A} \backslash\left\{\ell_{n}\right\}$, and denote $n^{\prime}:=n-1=\left|\mathcal{A}^{\prime}\right|$.

We can rewrite

$$
\mathbf{P}=\sum_{1 \leq i<j \leq n-1}\left[\left(\ell_{n} h_{i, j}+\frac{1}{2} h_{i, n} \ell_{j}+\frac{1}{2} h_{j, n} \ell_{i}\right) \prod_{v \in[n-1] \backslash\{i, j\}} \ell_{v}\right] .
$$

Since $I_{n-1}(\mathcal{A})=\ell_{n} I_{n^{\prime}-1}\left(\mathcal{A}^{\prime}\right)+I_{n^{\prime}}\left(\mathcal{A}^{\prime}\right) \subset I_{n^{\prime}-1}\left(\mathcal{A}^{\prime}\right)$, we have that

$$
\phi_{\mathcal{A}^{\prime}}\left(\ldots, \ell_{n} \widehat{h}_{i, j}, \ldots\right)=0,
$$

where the argument of the map has $\left(\begin{array}{c}n-1 \\ 2\end{array}\right)$ entries, as $1 \leq i<j \leq n-1$.

If the rank 2 flat $X:=V\left(\ell_{1}, \ell_{2}\right)$ has $\left|\mathcal{A}_{X}\right|=2$ (i.e., there is no 3-dependency of $\mathcal{A}$ containing both $\ell_{1}$ and $\left.\ell_{2}\right)$, then also $\left|\mathcal{A}_{X}^{\prime}\right|=2$. By induction, $\operatorname{ker}\left(\phi_{\mathcal{A}^{\prime}}\right)=\Lambda_{3}\left(\mathcal{A}^{\prime}\right)$, so $\ell_{n} \widehat{h}_{1,2}=0$ in $R /\left\langle\ell_{1}, \ell_{2}\right\rangle$. Since $\ell_{n} \notin\left\langle\ell_{1}, \ell_{2}\right\rangle$, we obtain $\widehat{h}_{1,2}=0$ in $R /\left\langle\ell_{1}, \ell_{2}\right\rangle$.

Suppose the flat $X=V\left(\ell_{1}, \ell_{2}\right)$ from above has $\mathcal{A}_{X}=\left\{\ell_{1}, \ell_{2}, \ldots, \ell_{s}\right\} \subset \mathcal{A}$ with $s \geq 3$. Since $\ell_{n} \notin\left\langle\ell_{1}, \ell_{2}\right\rangle$, we have again that $\left.\mathcal{A}_{X}^{\prime}=\mathcal{A}_{X} \subseteq \mathcal{A}^{\prime}\right]$

By induction, together with Claim 1, we have that the $\left(\begin{array}{l}s \\ 2\end{array}\right)$-tuple

$$
\left(\ell_{n} \widehat{h}_{1,2}, \ldots, \ell_{n} \widehat{h}_{i, j}, \ldots, \ell_{n} \widehat{h}_{s-1, s}\right) \in \Lambda_{3}\left(\mathcal{A}_{X}^{\prime}\right) .
$$

Same as in the proof of Claim 1, after a change of variables, let us assume that $\ell_{1}=x_{1}$, and $\ell_{i}=$ $x_{1}+\lambda_{i} x_{2}, \lambda_{i} \in \mathbb{K} \backslash\{0\}, i=2, \ldots, s$, and $\lambda_{i} \neq \lambda_{j}$, if $i \neq j$. Then

$$
\left(\ell_{n} \widehat{h}_{1,2}, \ldots, \ell_{n} \widehat{h}_{i, j}, \ldots, \ell_{n} \widehat{h}_{s-1, s}\right)=\sum_{2 \leq u<v \leq s} \widehat{g}_{u, v} \mathbf{c}_{1, u, v},
$$

for some $\widehat{g}_{u, v} \in R /\left\langle\ell_{1}, \ell_{2}\right\rangle \sqrt{2}$, and where for $2 \leq u<v \leq s$,

$$
\mathbf{c}_{1, u, v}=(0, \ldots, 0, \underbrace{-\lambda_{u}}_{(1, u)}, 0, \ldots, 0, \underbrace{\lambda_{v}}_{(1, v)}, 0, \ldots, 0,(\underbrace{\left(\lambda_{u}-\lambda_{v}\right)}_{(u, v)}, 0, \ldots, 0) .
$$

Equating each entry, we obtain that in $R /\left\langle\ell_{1}, \ell_{2}\right\rangle$ we have

\footnotetext{
${ }^{1}$ It is also clear, that for any $a \neq b \in\{1, \ldots, s\}$, we can pick $u(a, b)=n$.

${ }^{2}$ For any $u \neq v \in\{1, \ldots, s\}$, we have $\left\langle\ell_{u}, \ell_{v}\right\rangle=\left\langle\ell_{1}, \ell_{2}\right\rangle=\left\langle x_{1}, x_{2}\right\rangle$.
} 
(1) For each $2 \leq u<v \leq s$,

$$
\ell_{n} \widehat{h}_{u, v}=\left(\lambda_{u}-\lambda_{v}\right) \widehat{g}_{u, v}
$$

(2) For each $2 \leq w \leq s$,

$$
\ell_{n} \widehat{h}_{1, w}=\lambda_{w}\left(\sum_{t=2}^{w-1} \widehat{g}_{t, w}-\sum_{t=w+1}^{s} \widehat{g}_{w, t}\right) .
$$

Solving for $\widehat{g}_{u, v}$ in the first group of equations, and plugging in the second group of equations, we obtain that for each $2 \leq w \leq s$

$$
\ell_{n} \widehat{h}_{1, w}=\ell_{n} \lambda_{w}\left(\sum_{t=2}^{w-1} \frac{1}{\lambda_{t}-\lambda_{w}} \widehat{h}_{t, w}-\sum_{t=w+1}^{s} \frac{1}{\lambda_{w}-\lambda_{t}} \widehat{h}_{w, t}\right),
$$

which we can immediately simplify by $\ell_{n}$, since $\ell_{n} \notin\left\langle\ell_{1}, \ell_{2}\right\rangle$.

This lead to the following equation:

$$
\left(\widehat{h}_{1,2}, \ldots, \widehat{h}_{i, j}, \ldots, \widehat{h}_{s-1, s}\right)=\sum_{2 \leq u<v \leq s} \frac{\widehat{h}_{u, v}}{\lambda_{u}-\lambda_{v}} \mathbf{c}_{1, u, v} .
$$

One should observe that $\ell_{n}$ does not show up anywhere in this equation. What show up are all the pairs of distinct indices of the linear forms of $\mathcal{A}_{X}$, where $X=V\left(\ell_{u}, \ell_{v}\right)$, for any $u \neq v \in\{1, \ldots, s\}$.

The two cases above mean that $\operatorname{ker}\left(\phi_{\mathcal{A}}\right)$ is isomorphic to the direct sum of $\Lambda_{3}\left(\mathcal{A}_{X}\right)$, where $X$ scans over all rank 2 flats of $\mathcal{A}$ with $\left|\mathcal{A}_{X}\right| \geq 3$. With Claim 1, this proves the desired equality

$$
\operatorname{ker}\left(\phi_{\mathcal{A}}\right)=\Lambda_{3}(\mathcal{A})
$$

Before concluding this subsection, it is worth observing the following facts. If $X$ is a rank 2 flat with $\left|\mathcal{A}_{X}\right|=2$, then obviously $\Lambda_{3}\left(\mathcal{A}_{X}\right)=0$. So we showed that $\operatorname{ker}\left(\phi_{\mathcal{A}}\right) \cong \bigoplus_{X \in L_{2}(\mathcal{A})} \operatorname{ker}\left(\phi_{\mathcal{A}_{X}}\right)$, and from Claims 2 and 3, each direct summand is isomorphic to $\Lambda_{3}\left(\mathcal{A}_{X}\right)$.

In fact we obtained that the $R$-module $\operatorname{ker}\left(\phi_{\mathcal{A}}\right)$ "decomposes by localizations". The prime ideals $\mathfrak{p}_{i, j}:=$ $\left\langle\ell_{i}, \ell_{j}\right\rangle$ are the associated primes of the $R$-module $\Lambda(\mathcal{A})$. By localization we have two cases:

(1) If $\mathfrak{p}_{i, j} \neq \mathfrak{p}_{u, v}$, then $\mathfrak{p}_{u, v} R_{\mathfrak{p}_{i, j}}=R_{\mathfrak{p}_{i, j}}$, and therefore

$$
\left(\frac{R}{\mathfrak{p}_{u, v}}\right)_{\mathfrak{p}_{i, j}}=0 .
$$

(2) If $\mathfrak{p}_{i, j}=\mathfrak{p}_{u, v}$, then $\mathfrak{p}_{u, v} R_{\mathfrak{p}_{i, j}}=\mathfrak{p}_{i, j} R_{\mathfrak{p}_{i, j}}$, and therefore

$$
\left(\frac{R}{\mathfrak{p}_{u, v}}\right)_{\mathfrak{p}_{i, j}} \simeq \mathbb{K}
$$

as $R$-modules.

From the proof above, it becomes transparent that by localization at a prime ideal defining a rank 2 flat $X:=V\left(\mathfrak{p}_{i, j}\right)$, one obtains $\operatorname{ker}\left(\phi_{\mathcal{A}_{X}}\right)$, and therefore the decomposition

$$
\operatorname{ker}\left(\phi_{\mathcal{A}}\right) \simeq \bigoplus_{\mathfrak{p} \in A s_{R}(\Lambda(\mathcal{A}))} \operatorname{ker}\left(\phi_{\mathcal{A}}\right)_{\mathfrak{p}}
$$


If for any hyperplane arrangement $\mathcal{A} \subset R$, the $R$-module $R / I_{n-1}(\mathcal{A})$ is (arithmetically) CohenMacaulay (see [15, Lemma 3.2]), by comparison, that is the case for $R / I_{n-2}(\mathcal{A})$ only in special cases.

Corollary 2.5. Let $\mathcal{A} \subset R$ be a hyperplane arrangement with $|\mathcal{A}|=n \geq 2$. Then $R / I_{n-2}(\mathcal{A})$ is (arithmetically) Cohen-Macaulay if and only if either

(1) if $\operatorname{rank}(\mathcal{A})=2$, then $p(\mathcal{A})=\left(\begin{array}{c}n-1 \\ 2\end{array}\right)$, or

(2) if $\operatorname{rank}(\mathcal{A}) \geq 3$, then $p(\mathcal{A})=0$ (i.e., $\mathcal{A}$ is 3-generic, or any three linear forms of $\mathcal{A}$ are linearly independent).

Proof. From the minimal free resolution exhibited in Theorem 2.4, the projective dimension is

$$
\operatorname{pdim}_{R}\left(R / I_{n-2}(\mathcal{A})\right)= \begin{cases}2, & \text { if } p(\mathcal{A})=\left(\begin{array}{c}
n-1 \\
2
\end{array}\right) \\
3, & \text { otherwise. }\end{cases}
$$

From proof of Claim 3, if $\operatorname{rank}(\mathcal{A})=2$, then $p(\mathcal{A})=\left(\begin{array}{c}n-1 \\ 2\end{array}\right)$. For the converse, let $X_{1}, \ldots, X_{r}$ be all the distinct rank 2 flats of $L(\mathcal{A})$, and suppose $\left|\mathcal{A}_{X_{u}}\right|=n_{u}$, for $u=1, \ldots, r$. Obviously, $n_{u} \geq 2$, and from Claim 1

$$
\sum_{u=1}^{r}\left(\begin{array}{c}
n_{u}-1 \\
2
\end{array}\right)=\left(\begin{array}{c}
n-1 \\
2
\end{array}\right) .
$$

Also, for any $1 \leq i<j \leq n$, we have that $V\left(\ell_{i}, \ell_{j}\right)$ is a rank 2 flat of $\mathcal{A}$, and therefore

$$
\sum_{u=1}^{r}\left(\begin{array}{c}
n_{u} \\
2
\end{array}\right)=\left(\begin{array}{l}
n \\
2
\end{array}\right) \text {. }
$$

Subtracting these two equations one obtains

$$
n_{1}+\cdots+n_{r}-r=n-1 .
$$

Modulo the Claim 4 below, this is true only when $\operatorname{rank}(\mathcal{A})=2$ (in this case $r=1$ and $n_{1}=n$ ).

-Claim 4: If $\operatorname{rank}(\mathcal{A}) \geq 3$, then $n_{1}+\cdots+n_{r}-r \geq n$.

Proof of Claim 4. We will use induction on $|\mathcal{A}|=n \geq 3$. The base case, $n=3$, is immediate, since $\mathcal{A}$ will consist of three linearly independent linear forms, and so $r=3$, and $n_{1}=n_{2}=n_{3}=2$; indeed, $2+2+2-3=3 \geq 3$.

For the induction step, suppose $|\mathcal{A}| \geq 4$, and let $\mathcal{A}^{\prime}:=\mathcal{A} \backslash\left\{\ell_{n}\right\}$. Suppose that for some $1 \leq b \leq r$, $X_{i} \subset V\left(\ell_{n}\right)$ for $1 \leq i \leq b$, and $X_{j} \nsubseteq V\left(\ell_{n}\right)$ for $b+1 \leq j \leq r$. Also suppose for some $0 \leq a \leq b, n_{u}=2$ for $0 \leq u \leq a$, and $n_{v} \geq 3$ for $a+1 \leq v \leq b$.

If $\operatorname{rank}\left(\mathcal{A}^{\prime}\right) \geq 3$, then by induction, the claimed inequality is true for $\mathcal{A}^{\prime}$, so we have

$$
\left(n_{a+1}-1\right)+\cdots+\left(n_{b}-1\right)+n_{b+1}+\cdots+n_{r}-(r-a) \geq n-1,
$$

which give

$$
2 a+n_{a+1}+\cdots+n_{r}-r \geq n-1+b .
$$

Since $n_{1}+\cdots+n_{a}=2 a$, and $b \geq 1$, the method of induction proves the inequality.

If $\operatorname{rank}\left(\mathcal{A}^{\prime}\right)=2$, then, $\operatorname{since} \operatorname{rank}(\mathcal{A})=3$, after a change of variables we have that in $\mathbb{P}^{2}, \mathcal{A}^{\prime}$ is a pencil of $n-1$ lines through a point, and $\ell_{n}$ is a line that misses that point. So $r=n$, and $n_{1}=n-1$, and $n_{i}=2$ for $2 \leq i \leq n$. With this, we have

$$
n_{1}+\cdots+n_{r}-r=(n-1)+2(n-1)-n=2 n-3 \geq n .
$$

From the beginning of [18, Section 2], any minimal prime of $I_{n-2}(\mathcal{A})$ is of the form $\left\langle\ell_{u}, \ell_{v}, \ell_{w}\right\rangle$, for some (any) $1 \leq u<v<w \leq n$. So ht $\left(I_{n-2}(\mathcal{A})\right)=3$, if and only if $\Lambda_{3}(\mathcal{A})=0$. But this is equivalent to $p(\mathcal{A})=0$. 
2.3.2. The case of three variables, $k=3$. Let $\mathcal{A}=\left\{\ell_{1}, \ldots, \ell_{n}\right\} \subset R:=\mathbb{K}[x, y, z]$ be a line arrangement in $\mathbb{P}^{2}$, and suppose $\operatorname{rank}(\mathcal{A})=3$. Let $\mathfrak{m}:=\langle x, y, z\rangle$. Then, all the flats of $\operatorname{rank} 2$ in $L(\mathcal{A})$ correspond to the points of intersection among the lines of $\mathcal{A}$, say $P_{1}, \ldots, P_{s}$ (distinct). Often, this set is denoted $\operatorname{Sing}(\mathcal{A})$, and it is called the singularity locus of $\mathcal{A}$.

For $i=1, \ldots, s$, let $n_{i}:=\left|\mathcal{A}_{P_{i}}\right|$ be the number of lines of $\mathcal{A}$ intersecting at $P_{i}$.

Lemmas 3.1 and 3.2 in [15] say that $I_{n-1}(\mathcal{A})$ has primary decomposition

$$
I_{n-1}(\mathcal{A})=I\left(P_{1}\right)^{n_{1}-1} \cap \cdots \cap I\left(P_{s}\right)^{n_{s}-1} .
$$

In this section we are interested in finding a similar primary decomposition, but for $I_{n-2}(\mathcal{A})$.

First suppose that $\mathcal{A}$ is generic (i.e., any three of the linear forms of $\mathcal{A}$ are linearly independent). Let $\mathcal{C}_{\mathcal{A}}$ be the linear code with generating matrix $G$ having columns dual to the linear forms defining $\mathcal{A}$. This code has length $n$ and dimension 3. Since any three of the linear forms of $\mathcal{A}$ are linearly independent, the maximum number of columns of $G$ that span a $3-1=2$ dimensional vector space is 2 . So the minimum distance of $\mathcal{C}_{\mathcal{A}}$ is $n-2$ (see, e.g., [20, Remark 2.2]). But in these condition, by [18, Theorem 3.1], we have that $I_{n-2}(\mathcal{A})=\mathfrak{m}^{n-2}$, which is primary.

Suppose $\mathcal{A}$ is not generic. Then $\operatorname{ht}\left(I_{n-2}(\mathcal{A})\right)=2$. Since $\operatorname{ht}\left(I_{n-1}(\mathcal{A})\right)=2$, and $\operatorname{ht}\left(I_{n}(\mathcal{A})\right)=1$, by Propositions 2.2 and 2.3 in [1], we have

$$
I_{n-2}(\mathcal{A})=I\left(P_{1}\right)^{n_{1}-2} \cap \cdots \cap I\left(P_{s}\right)^{n_{s}-2} \cap K,
$$

where $K$ is $\mathfrak{m}$-primary ideal. Also, for any ideal $I \subset R$, by convention $I^{0}=R$.

Let $I$ be an ideal of $R$. Then the saturation of $I$ is the ideal

$$
I^{\text {sat }}:=\left\{f \in R \mid \mathfrak{m}^{v_{f}} \cdot f \in I, \text { for some integer } v_{f} \geq 0\right\} .
$$

Obviously, $I \subseteq I^{\text {sat }}$.

Since $\sqrt{K}=\mathfrak{m}$, there exists an integer $w \geq 1$, such that $\mathfrak{m}^{w} \subseteq K$. So $K^{\text {sat }}=R$. For any $j=1, \ldots, s$, $I\left(P_{j}\right)$ is a linear prime ideal of height 2 . So there exists one generator $L_{j}$ of $\mathfrak{m}$ that does not belong to $I\left(P_{j}\right)$. But then, $I\left(P_{j}\right)^{n_{j}-2}: L_{j}=I\left(P_{j}\right)^{n_{j}-2}$, giving that $\left(I\left(P_{j}\right)^{n_{j}-2}\right)^{s a t}=I\left(P_{j}\right)^{n_{j}-2}$. These, together with the formula $(I \cap J)^{\text {sat }}=I^{\text {sat }} \cap J^{\text {sat }}$, give

$$
I_{n-2}(\mathcal{A})^{s a t}=I\left(P_{1}\right)^{n_{1}-2} \cap \cdots \cap I\left(P_{s}\right)^{n_{s}-2} .
$$

For any ideal $I$ of $R$, by definition,

$$
\mathrm{H}_{\mathfrak{m}}^{0}(R / I)=\left\{\hat{f} \in R / I \mid \mathfrak{m}^{v_{f}} \cdot f \in I\right\},
$$

so $\mathrm{H}_{\mathfrak{m}}^{0}(R / I)=I^{\text {sat }} / I$.

Denote, $M:=\mathrm{H}_{\mathfrak{m}}^{0}\left(R / I_{n-2}(\mathcal{A})\right)$. By Theorem 2.4 $\operatorname{reg}\left(R / I_{n-2}(\mathcal{A})\right)=n-3$. Therefore, by Corollaries 4.5 and 4.4 in [6], $\max \left\{d \mid M_{d} \neq 0\right\} \leq n-3$, which leads to

$$
\left(I_{n-2}(\mathcal{A})^{s a t} / I_{n-2}(\mathcal{A})\right)_{e}=0, \text { for any } e \geq n-2 .
$$

This means that

$$
I_{n-2}(\mathcal{A})=I_{n-2}(\mathcal{A})^{\text {sat }} \cap \mathfrak{m}^{n-2} .
$$

Considering that if $\mathcal{A}$ is generic, then $n_{j}=2$ for all $j=1, \ldots, s$, putting everything together we have the following result

Proposition 2.6. Let $\mathcal{A}$ be an essential line arrangement in $\mathbb{P}^{2}$. Suppose $|\mathcal{A}|=n$, and $\operatorname{Sing}(\mathcal{A})=$ $\left\{P_{1}, \ldots, P_{s}\right\}$, with $n_{j}=\left|\mathcal{A}_{P_{j}}\right|, j=1, \ldots, s$. Then, in $R=\mathbb{K}[x, y, z]$, we have the primary decomposition

$$
I_{n-2}(\mathcal{A})=I\left(P_{1}\right)^{n_{1}-2} \cap \cdots \cap I\left(P_{s}\right)^{n_{s}-2} \cap\langle x, y, z\rangle^{n-2} .
$$




\section{ORLIK-TERAO ALGEBRA OF THE SECOND ORDER}

Let $\mathcal{A}=\left\{\ell_{1}, \ldots, \ell_{n}\right\} \subset R:=\mathbb{K}\left[x_{1}, \ldots, x_{k}\right]$. According to [10, Example 2.2(iii)], for $S_{i, j}:=[n] \backslash$ $\{i, j\}, 1 \leq i<j \leq n$, and $\mathfrak{S}:=\left\{\ldots, S_{i, j}, \ldots\right\}$, we define the Orlik-Terao algebra of the second order of $\mathcal{A}$ to be

$$
O T(2, \mathcal{A}):=O T(\mathfrak{S}, \mathcal{A})=\mathbb{K}\left[\ldots, \frac{1}{\ell_{i} \ell_{j}}, \ldots\right] .
$$

In this section we study the first properties of this algebra, also making some links, if they exist, with $O T(\mathcal{A})$, the (classical) Orlik-Terao algebra of $\mathcal{A}$.

From [10, Proposition 2.3 and Theorem 2.4], with the notations at the beginning of Section 2, i.e., $f:=$ $\ell_{1} \cdots \ell_{n}$, and $f_{i, j}:=f /\left(\ell_{i} \ell_{j}\right), 1 \leq i<j \leq n$, we have the following isomorphisms of graded $\mathbb{K}$-algebra

$$
O T(2, \mathcal{A}) \simeq \mathbb{K}\left[\ldots, f_{i, j}, \ldots\right] \simeq \mathcal{F}\left(I_{n-2}(\mathcal{A})\right),
$$

where $\mathcal{F}\left(I_{n-2}(\mathcal{A})\right)$ is the special fiber of the ideal $I_{n-2}(\mathcal{A})$.

Denote $\mathbf{T}:=\mathbb{K}\left[\ldots, t_{i, j}, \ldots\right], 1 \leq i<j \leq n$. Because of the above isomorphism with the special fiber, the defining ideal of $O T(2, \mathcal{A})$ is

$$
I(2, \mathcal{A}):=\left\{F \in \mathbf{T} \mid F\left(\ldots, f_{i, j}, \ldots\right)=0\right\},
$$

and so $O T(2, \mathcal{A}) \simeq \mathbf{T} / I(2, \mathcal{A})$.

Proposition 3.1. If $\operatorname{rank}(\mathcal{A})=k \geq 2$ (i.e., $\mathcal{A}$ is essential), then the Krull dimension of $O T(2, \mathcal{A})$, and therefore the analytic spread of $I_{n-2}(\mathcal{A})$, equals $k$.

Proof. We have $O T(2, \mathcal{A})=\mathbb{K}\left[\ldots, \frac{1}{\ell_{i} \ell_{j}}, \ldots\right]$, which is an integral domain. Then the total field of fractions is $Q(O T(2, \mathcal{A}))=\mathbb{K}\left(\ldots, \ell_{i} \ell_{j}, \ldots\right)$.

Since $\mathcal{A}$ is essential, after a change of variables we can suppose that $\ell_{i}=x_{i}$, for $i=1, \ldots, k$. We have the following sequence of inclusions:

$$
\mathbb{K}\left(\ldots, x_{i} x_{j}, \ldots\right) \subseteq Q(O T(2, \mathcal{A})) \subset \mathbb{K}\left(x_{1}, \ldots, x_{k}\right),
$$

where the left-most field is the field of fraction of $\mathbb{K}[G]$, where $G$ is the complete graph on $k$ vertices. By [22, Corollary 10.1.21], the Krull dimension of $\mathbb{K}[G]$ is $k$, and therefore tr. $\operatorname{deg}_{\mathbb{K}} \mathbb{K}\left(\ldots, x_{i} x_{j}, \ldots\right)=k$. Since tr. $\operatorname{deg}_{\mathbb{K}} \mathbb{K}\left(x_{1}, \ldots, x_{k}\right)=k$, we obtain

$$
\operatorname{tr} \cdot \operatorname{deg}_{\mathbb{K}} Q(O T(2, \mathcal{A}))=k,
$$

and hence, the result.

Since $I_{n-2}(\mathcal{A})$ is linearly presented, an immediate consequence of Proposition 3.1, via [4, Theorem 3.2], and under the assumption that $\mathcal{A}$ is essential, is that the rational map

$$
\mathbb{P}^{k-1} \rightarrow \mathbb{P}^{m-1},\left[x_{1}, \ldots, x_{k}\right] \mapsto\left[\ldots, 1 /\left(\ell_{i} \ell_{j}\right), \ldots\right], 1 \leq i<j \leq n,
$$

where $m=\left(\begin{array}{l}n \\ 2\end{array}\right)$, is birational onto its image.

For the remainder of this article, we focus our attention on the generators of $I(2, \mathcal{A})$. First, we have the following lemma.

Lemma 3.2. The generators of $I(2, \mathcal{A})$ include the following standard elements: 
(1) LINEAR: If $c_{i_{1}} \ell_{i_{1}}+c_{i_{2}} \ell_{i_{2}}+c_{i_{3}} \ell_{i_{3}}=0,1 \leq i_{1}<i_{2}<i_{3} \leq n$, is a 3-dependency, then

$$
\underbrace{c_{i_{1}} t_{i_{2}, i_{3}}+c_{i_{2}} t_{i_{1}, i_{3}}+c_{i_{3}} t_{i_{1}, i_{2}}}_{L_{i_{1}, i_{2}, i_{3}}} \in I(2, \mathcal{A}) .
$$

(2) QUADRATIC: If $n \geq 4$, then for any $1 \leq u<v<w<z \leq n$

$$
\underbrace{t_{u, v} t_{w, z}-t_{u, w} t_{v, z}}_{Q_{u, v, w, z}^{1}}, \underbrace{t_{u, v} t_{w, z}-t_{u, z} t_{v, w}}_{Q_{u, v, w, z}^{2}} \in I(2, \mathcal{A}) .
$$

Proof. The linear generators are obtained from multiplying the dependency by $\prod_{j \in[n] \backslash\left\{i_{1}, i_{2}, i_{3}\right\}} \ell_{j}$, obtaining $c_{i_{1}} f_{i_{2}, i_{3}}+c_{i_{2}} f_{i_{1}, i_{3}}+c_{i_{3}} f_{i_{1}, i_{2}}=0$.

The quadratic generators are obtained from the commutativity of the products of linear forms.

Denote $\mathbf{S}:=\mathbb{K}\left[y_{1}, \ldots, y_{n}\right]$, and denote $I(\mathcal{A})$ to be the defining ideal of the (usual) Orlik-Terao algebra, i.e., $O T(\mathcal{A}) \simeq \mathbf{S} / I(\mathcal{A})$.

We have the following first result.

Proposition 3.3. The map

$$
O T(2, \mathcal{A}) \longrightarrow O T(\mathcal{A}), t_{i, j} \mapsto y_{i} y_{j}
$$

is a well-defined embedding of algebras.

Proof. First we show "well-defined". Let $F \in I(2, \mathcal{A})$ be homogeneous in the $t_{i, j}$ variables, of degree $d$. Then

$$
F\left(\ldots, f_{i, j}, \ldots\right)=F\left(\ldots, \frac{f}{\ell_{i} \ell_{j}}, \ldots\right)=0 .
$$

Multiplying this by $f^{d}$, and distributing $f$ with the appropriate powers, we obtain

$$
F\left(\ldots, \frac{f}{\ell_{i}} \cdot \frac{f}{\ell_{j}}, \ldots\right)=0,
$$

which means that $F\left(\ldots, y_{i} y_{j}, \ldots\right) \in I(\mathcal{A}) \subset \mathbf{S}$.

The "embedding" part of the statement follows the reverse argument: let $F \in \mathbf{T}$ be homogeneous in the $t_{i, j}$ variables, of degree $d$, such that $F\left(\ldots, y_{i} y_{j}, \ldots\right) \in I(\mathcal{A})$. Then,

$$
F\left(\ldots, \frac{f}{\ell_{i}} \cdot \frac{f}{\ell_{j}}, \ldots\right)=0 .
$$

Taking $f^{d}$ common factor from all the terms, after regrouping we obtain

$$
F\left(\ldots, \frac{f}{\ell_{i} \ell_{j}}, \ldots\right)=F\left(\ldots, f_{i, j}, \ldots\right)=0,
$$

hence $F \in I(2, \mathcal{A})$.

Observe that the linear elements obtained in Lemma 3.2, via the map in Proposition 3.3, give the wellknown quadratic elements $c_{i_{1}} y_{i_{2}} y_{i_{3}}+c_{i_{2}} y_{i_{1}} y_{i_{3}}+c_{i_{3}} y_{i_{1}} y_{i_{2}}$ of $I(\mathcal{A})$. The quadratic generators obtained there give only that $0 \in I(\mathcal{A})$.

Let $J(\mathcal{A})$ be the ideal of $\mathbf{S}$, defined as:

$$
J(\mathcal{A}):=\left\{F\left(\ldots, y_{i} y_{j}, \ldots\right) \mid F \in I(2, \mathcal{A}) \subset \mathbf{T}\right\} .
$$

From Proposition 3.3 we have $J(\mathcal{A}) \subseteq I(\mathcal{A})$.

Consider the subring $\mathbf{S}^{\prime}:=\mathbb{K}\left[\ldots, y_{i} y_{j}, \ldots\right] \subset \mathbf{S}$, and let $J^{\prime}(\mathcal{A}):=J(\mathcal{A}) \cap \mathbf{S}^{\prime}$. Naturally, $\mathbf{S}^{\prime}$ is isomorphic as a graded $\mathbb{K}$-algebra to $\mathbf{T}$ quotient by the standard quadratic generators presented in Lemma 3.2 .

Proposition 3.4. We have the following properties: 
(1) In $\mathbf{S}$, one has $J(\mathcal{A}):\left\langle y_{1}, \ldots, y_{n}\right\rangle=I(\mathcal{A})$.

(2) $J^{\prime}(\mathcal{A})=I(\mathcal{A}) \cap \mathbf{S}^{\prime}$.

(3) $O T(2, \mathcal{A}) \simeq \frac{\mathbf{S}^{\prime}}{J^{\prime}(\mathcal{A})}$.

Proof. To prove (1), suppose $c_{i_{1}} \ell_{i_{1}}+\cdots+c_{i_{s}} \ell_{i_{s}}=0$ is a minimal dependency in $\mathcal{A}$. This leads to the generator

$$
G_{i_{1}, \ldots, i_{s}}:=c_{i_{1}} y_{i_{2}} \cdots y_{i_{s}}+\cdots+c_{i_{s}} y_{i_{1}} \cdots y_{i_{s-1}}
$$

of $I(\mathcal{A})$ (see [13]). We have two cases:

- If $s=2 a+1$, then $s-1$ is even, and in each term we can group pairs of $y$ 's with different indices to obtain an element of $I(2, \mathcal{A})$ of degree $a=(s-1) / 2$.

- If $s=2 a$, then $s-1$ is odd. But we can multiply this generator of $I(\mathcal{A})$, by any $y_{1}, \ldots, y_{n}$, to obtain via pairings similar as above (of course, making sure that if $y_{j}$ shows twice in a term, we don't pair it with itself), to obtain elements of $I(2, \mathcal{A})$, of degree $a$.

We can see that a generator of $I(\mathcal{A})$ either by itself is in $J(\mathcal{A})$, or multiplied by a variable $y_{j}$; this gives the inclusion " $\supseteq$ ". For the inclusion " $\subseteq$ ", if $y_{j} \cdot F \in J(\mathcal{A}) \subseteq I(\mathcal{A})$, and since $I(\mathcal{A})$ is non-degenerate prime ideal (see [16, Corollary 2.2]), then $F \in I(\mathcal{A})$.

The last two statements are immediate from Proposition 3.3 .

If $n=3$ and $\operatorname{rank}(\mathcal{A})=3$, or if $n=2$ and $\operatorname{rank}(\mathcal{A})=2$, since $I(\mathcal{A})=0$ in both cases, then $I(2, \mathcal{A})=0$ as well in both cases (we have $n<4$ ).

3.1. Generators of the ideal of the Orlik-Terao algebra of the second order. In the proof of Proposition 3.4 we give a glimpse of a standard way to find elements of $I(2, \mathcal{A})$, that together with the elements obtained in Lemma 3.2 will form a generating set for $I(2, \mathcal{A})$.

Let us consider again the element $G_{i_{1}, \ldots, i_{s}} \in I(\mathcal{A})$ corresponding to the circuit $\left\{i_{1}, \ldots, i_{s}\right\}, s \geq 3$. The whole idea is to multiply $G_{i_{1}, \ldots, i_{s}}$ by a convenient monomial $M \in \mathbf{S}$ (possibly 1 ) such that in each term of this product, to be able to pair any two $y$ 's with distinct indices (i.e., $M G_{i_{1}, \ldots, i_{s}} \in \mathbf{S}^{\prime}$ ). Obviously $\operatorname{deg}(M)+s-1$ must be an even number, and if variable $y_{a}$ shows up in a term of $M G_{i_{1}, \ldots, i_{s}}$ with exponent $m_{a}$, since we cannot pair two $y_{a}$ 's together, we must have $m_{a} \leq$ than the sum of the exponents of all the other variables in that term.

Often there will be a multitude of possible pairings, but the quadratic elements obtained in Lemma 3.2 will help consider fewer. Nonetheless, as one can see in the example below, to find efficient ways to choose those monomials $M$ that will lead only to minimal generators of $I(2, \mathcal{A})$, becomes a delicate technical problem.

Example 3.5. Let $\mathcal{A}=\left\{\ell_{1}, \ell_{2}, \ell_{3}, \ell_{4}\right\} \subset \mathbb{K}\left[x_{1}, x_{2}, x_{3}\right]$, with

$$
\ell_{1}=x_{1}, \ell_{2}=x_{2}, \ell_{3}=x_{1}+x_{2}, \ell_{4}=x_{3} .
$$

We have $G:=G_{1,2,3}=y_{2} y_{3}+y_{1} y_{3}-y_{1} y_{2} \in I(\mathcal{A}) \subset \mathbf{S}=\mathbb{K}\left[y_{1}, y_{2}, y_{3}, y_{4}\right]$, and the standard generators of $I(2, \mathcal{A}) \subset \mathbf{T}=\mathbb{K}\left[t_{1,2}, \ldots, t_{3,4}\right]$ exhibited in Lemma 3.2 are:

$$
\begin{aligned}
L_{1,2,3} & =t_{2,3}+t_{1,3}-t_{1,2} \\
Q_{1,2,3,4}^{1} & =t_{1,2} t_{3,4}-t_{1,3} t_{2,4} \\
Q_{1,2,3,4}^{2} & =t_{1,2} t_{3,4}-t_{1,4} t_{2,3} .
\end{aligned}
$$

Let $M=y_{1}^{m_{1}} y_{2}^{m_{2}} y_{3}^{m_{3}} y_{4}^{m_{4}}$ be the monomial such that $M G \in \mathbf{S}^{\prime}=\mathbb{K}\left[y_{1} y_{2}, \ldots, y_{3} y_{4}\right]$. Let $d:=m_{1}+$ $m_{2}+m_{3}+m_{4}$. Then, $d$ must be an even number. We also must have

$$
2 m_{1} \leq d, 2 m_{2} \leq d, 2 m_{3} \leq d, 2 m_{4} \leq d+2 .
$$

- If $m_{4}=0$, then $d=m_{1}+m_{2}+m_{3}$, with $m_{1} \leq m_{2}+m_{3}, m_{2} \leq m_{1}+m_{3}$, and $m_{3} \leq m_{1}+m_{2}$. So $M \in \mathbf{S}^{\prime}$. Suppose $N$ is the preimage of $M$ in $\mathbf{T}$. Since we need a new generator, therefore different 
than $N L_{1,2,3}$, the only way would be to take (if possible) two distinct variables from $M$ and pair them "differently" with the variables in each term of $G$. Since we can suppose that $m_{1} \geq 1$ and $m_{2} \geq 1$,

$$
M G=\left(y_{1} y_{2} y_{2} y_{3}+y_{1} y_{2} y_{1} y_{3}-y_{1} y_{2} y_{1} y_{2}\right) \frac{M}{y_{1} y_{2}},
$$

where the monomial $M /\left(y_{1} y_{2}\right)$ is assumed to be in $\mathbf{S}^{\prime}$. But it is clear that in parenthesis we obtain $t_{1,2} L_{1,2,3}$, so no new generator.

- Suppose $m_{4} \geq 1$ and $d=2$. If $m_{4}=2$, then $M G$ gives the new minimal generator

$$
t_{2,4} t_{3,4}+t_{1,4} t_{3,4}-t_{1,4} t_{2,4} .
$$

If $m_{4}=1$ and, say, $m_{1}=1$, then $M G=y_{1} y_{4} y_{2} y_{3}+y_{1} y_{4} y_{1} y_{3}-y_{1} y_{4} y_{1} y_{2}$. In the last two terms, since we cannot par $y_{1}$ with itself, we can only pair $y_{1} y_{4}, y_{1} y_{3}$, and $y_{1} y_{2}$. There are three different pairings we can do in the first term. But modulo the elements $Q_{1,2,3,4}^{1}$ and $Q_{1,2,3,4}^{2}$ we obtain $t_{1,4} L_{1,2,3}$, so no new minimal generator.

- Suppose $m_{4} \geq 1$. Suppose $d \geq 4$. Again, $M \in \mathbf{S}^{\prime}$, and let $N$ be its preimage in $\mathbf{T}$. Since we need a new generator, so different than $N L_{1,2,3}$, the only way would be to take (if possible) two variables from $M$ and pair them "differently" with the variables in each term of $G$. From the first two bullets, the two variables we pick should be $y_{4}$, and one of the other three. Since $d \geq 4$, then not all $m_{1}, m_{2}, m_{3}$ are zero. Suppose $m_{1} \geq 1$, and that we picked also $y_{1}$, and $M /\left(y_{1} y_{4}\right) \in \mathbf{S}^{\prime}$. But the second bullet tells us that we do not get a new minimal generator for $I(2, \mathcal{A})$.

In conclusion,

$$
I(2, \mathcal{A})=\left\langle L_{1,2,3}, Q_{1,2,3,4}^{1}, Q_{1,2,3,4}^{2}, t_{2,4} t_{3,4}+t_{1,4} t_{3,4}-t_{1,4} t_{2,4}\right\rangle .
$$

3.2. The symmetric ideal of $I_{n-2}(\mathcal{A})$ and Sylvester forms. Denote $\mathbb{T}:=R\left[\ldots, t_{i, j}, \ldots\right], 1 \leq i<j \leq$, with the natural bi-grading: $\operatorname{deg}\left(x_{u}\right)=(1,0)$, and $\operatorname{deg}\left(t_{i, j}\right)=(0,1)$. The Rees algebra of $I_{n-2}(\mathcal{A})$, namely $R\left[I_{n-2}(\mathcal{A}) t\right]$, is isomorphic as bi-graded algebras to $\mathbb{T} / \mathcal{I}(\mathcal{A}, n-2)$, for some ideal $\mathcal{I}(\mathcal{A}, n-2) \subset \mathbb{T}$, called the Rees ideal of $I_{n-2}(\mathcal{A})$, or the presentation ideal of $R\left[I_{n-2}(\mathcal{A}) t\right]$.

The symmetric ideal of $I_{n-2}(\mathcal{A})$ is the ideal of $\mathbb{T}$ generated by $\mathcal{I}(\mathcal{A}, n-2)_{(-, 1)}$, and it will be denoted here by $\operatorname{sym}\left(I_{n-2}(\mathcal{A})\right)$.

We have that $\operatorname{sym}\left(I_{n-2}(\mathcal{A})\right)$ is generated by the linear generators obtained in Lemma 3.2, and from Theorem 2.4, by the linear syzygies on the standard generators $f_{i, j}$ of $I_{n-2}(\mathcal{A})$.

For any $1 \leq a<b<c \leq n$ we have the standard syzygies $\ell_{a} f_{a, b}=\ell_{c} f_{b, c}, \ell_{a} f_{a, c}=\ell_{b} f_{b, c}$, and $\ell_{b} f_{a, b}=\ell_{c} f_{a, c}$, leading to

$$
\underbrace{\ell_{a} t_{a, b}-\ell_{c} t_{b, c}}_{A_{a, b, c}}, \underbrace{\ell_{a} t_{a, c}-\ell_{b} t_{b, c}}_{B_{a, b, c}}, \underbrace{\ell_{b} t_{a, b}-\ell_{c} t_{a, c}}_{C_{a, b, c}} \in \operatorname{sym}\left(I_{n-2}(\mathcal{A})\right) .
$$

Proposition 3.6. The symmetric ideal $\operatorname{sym}\left(I_{n-2}(\mathcal{A})\right)$ is generated by all $L_{i_{1}, i_{2}, i_{3}}$, whenever $\left\{i_{1}, i_{2}, i_{3}\right\}$ is a circuit, and by $A_{a, b, c}, B_{a, b, c}, C_{a, b, c}$, for all $1 \leq a<b<c \leq n$.

Proof. Let

$$
\sum_{1 \leq i<j \leq n} h_{i, j} f_{i, j}=0
$$

be a linear syzygy. Then the vector $\left(\ldots, \widehat{h}_{i, j}, \ldots\right) \in \operatorname{ker}\left(\phi_{\mathcal{A}}\right)$. By Lemma 2.3 and Claim 1 , this vector a combination of 3-dependencies, with coefficients linear forms. This means that this syzygy is a combination of the "syzygies" corresponding to the 3 -dependencies, modulo $R /\left\langle\ell_{i}, \ell_{j}\right\rangle$ in each $(i, j)$ entry.

Since we already accounted for the "syzygies" corresponding to the 3-dependencies, we can assume that each $h_{i, j} \in\left\langle\ell_{i}, \ell_{j}\right\rangle$. Suppose for all $1 \leq i<j \leq n$,

$$
h_{i, j}=\alpha_{i, j} \ell_{i}+\beta_{i, j} \ell_{j}, \alpha_{i, j}, \beta_{i, j} \in \mathbb{K} .
$$

These plugged back in the syzygy equation lead to a syzygy on $f_{1}:=f / \ell_{1}, \ldots, f_{n}:=f / \ell_{n}$, the generators of $I_{n-1}(\mathcal{A})$ 


$$
\sum_{i=1}^{n}\left(\sum_{u=1}^{i-1} \alpha_{u, i}+\sum_{v=i+1}^{n} \beta_{i, v}\right) f_{i}=0 .
$$

Since $\operatorname{gcd}\left(\ell_{i}, \ell_{j}\right)=1, i \neq j$, and since $\alpha_{i, j}, \beta_{i, j} \in \mathbb{K}$, we have that for each $i=1, \ldots, n$,

$$
\sum_{u=1}^{i-1} \alpha_{u, i}+\sum_{v=i+1}^{n} \beta_{i, v}=0 .
$$

With these equations we can rewrite the syzygy in the following way:

$$
\begin{array}{rcc}
\beta_{1,2}\left(\ell_{2} f_{1,2}-\ell_{n} f_{1, n}\right)+\beta_{1,3}\left(\ell_{3} f_{1,3}-\ell_{n} f_{1, n}\right)+ & \cdots & +\beta_{1, n-1}\left(\ell_{n-1} f_{1, n-1}-\ell_{n} f_{1, n}\right)+ \\
\alpha_{1,2}\left(\ell_{1} f_{1,2}-\ell_{n} f_{2, n}\right)+\beta_{2,3}\left(\ell_{3} f_{2,3}-\ell_{n} f_{2, n}\right)+ & \cdots & +\beta_{2, n-1}\left(\ell_{n-1} f_{2, n-1}-\ell_{n} f_{2, n}\right)+ \\
\vdots & \\
\alpha_{1, n}\left(\ell_{1} f_{1, n}-\ell_{n-1} f_{n-1, n}\right)+\alpha_{2, n}\left(\ell_{2} f_{2, n}-\ell_{n-1} f_{n-1, n}\right)+ & \cdots & +\alpha_{n-2, n}\left(\ell_{n-2} f_{n-2, n}-\ell_{n-1} f_{n-1, n}\right) .
\end{array}
$$

But this expression confirms that the syzygy can be written as a combination of the standard syzygies. So the result is shown.

Remark 3.7. In Theorem 2.4, the dimension of standard syzygies equals $2\left(\begin{array}{l}n \\ 2\end{array}\right)-n-2 p(\mathcal{A})=n(n-$ $2)-2 p(\mathcal{A})$. Since $p(\mathcal{A})$ is the number of minimal linear generators of $\operatorname{sym}\left(I_{n-2}(\mathcal{A})\right)$, we obtain that the minimum number of generators of $\operatorname{sym}\left(I_{n-2}(\mathcal{A})\right)$ is $n(n-2)-p(\mathcal{A})$.

3.2.1. Sylvester forms. The Sylvester forms technique (see for example [12, Section 2]) is a nice way to find new elements of the Rees ideal, from old elements. This technique was successfully applied in [10, Proposition 3.5], to obtain all the generators of $I(\mathcal{A})$, the Orlik-Terao ideal, and we will do the same to obtain elements in $I(2, \mathcal{A})$, from generators of $\operatorname{sym}\left(I_{n-2}(\mathcal{A})\right)$.

Since the level of computations exceeds the plans of this article, we are just going to exemplify them for some basic situations.

- Suppose $\ell_{3}=a_{1} \ell_{1}+a_{2} \ell_{2}$ is a 3-dependency; of course $\ell_{1}, \ell_{2}$ will form a regular sequence in $R$. If we consider $A_{1,2,3}=\ell_{1} t_{1,2}-\ell_{3} t_{2,3}=\ell_{1}\left(t_{1,2}-a_{1} t_{2,3}\right)+\ell_{2}\left(-a_{2} t_{2,3}\right)$ and $B_{1,2,3}=\ell_{1} t_{1,3}+\ell_{2}\left(-t_{2,3}\right)$, then we have the matrix equation

$$
\left[\begin{array}{l}
A_{1,2,3} \\
B_{1,2,3}
\end{array}\right]=\left[\begin{array}{cc}
t_{1,2}-a_{1} t_{2,3} & -a_{2} t_{2,3} \\
t_{1,3} & -t_{2,3}
\end{array}\right] \cdot\left[\begin{array}{l}
\ell_{1} \\
\ell_{2}
\end{array}\right] .
$$

Taking the determinant of the $2 \times 2$ content matrix we obtain

$$
-t_{2,3}\left(t_{1,2}-a_{1} t_{2,3}-a_{2} t_{1,3}\right)= \pm t_{2,3} L_{1,2,3}
$$

as an element of $I(2, \mathcal{A})$. Since $I(2, \mathcal{A})$ is prime, we obtain the linear generator $L_{1,2,3} \in I(2, \mathcal{A})$.

- Suppose $\ell_{4}=a_{1} \ell_{1}+a_{2} \ell_{2}+a_{3} \ell_{3}$ is a dependency, with $\ell_{1}, \ell_{2}, \ell_{3}$ being linearly independent. With this dependency, and choosing $A_{1,2,3}, B_{1,2,3}, A_{1,2,4}$ we have the matrix equation

$$
\left[\begin{array}{l}
A_{1,2,3} \\
B_{1,2,3} \\
A_{1,2,4}
\end{array}\right]=\left[\begin{array}{ccc}
t_{1,2} & 0 & -t_{2,3} \\
t_{1,3} & -t_{2,3} & 0 \\
t_{1,2}-a_{1} t_{2,4} & -a_{2} t_{2,4} & -a_{3} t_{2,4}
\end{array}\right] \cdot\left[\begin{array}{c}
\ell_{1} \\
\ell_{2} \\
\ell_{3}
\end{array}\right] .
$$

Taking the determinant of the $3 \times 3$ content matrix we obtain

$$
t_{2,3} \underbrace{\left(a_{3} t_{1,2} t_{2,4}+a_{2} t_{1,3} t_{2,4}+a_{1} t_{2,3} t_{2,4}-t_{1,2} t_{2,3}\right)}_{F} \in I(2, \mathcal{A}) .
$$


So $F \in I(2, \mathcal{A})$.

If $G=a_{1} y_{2} y_{3} y_{4}+a_{2} y_{1} y_{3} y_{4}+a_{3} y_{1} y_{2} y_{4}-y_{1} y_{2} y_{3}$ is the generators of $I(\mathcal{A})$ corresponding to the given dependency, then $F$ is the preimage in $\mathbf{T}$ of

$$
y_{2} G=a_{1}\left(y_{2} y_{3}\right)\left(y_{2} y_{4}\right)+a_{2}\left(y_{1} y_{3}\right)\left(y_{2} y_{4}\right)+a_{3}\left(y_{1} y_{2}\right)\left(y_{2} y_{4}\right)-\left(y_{1} y_{2}\right)\left(y_{2} y_{3}\right) .
$$

- Even the quadratic standard generators of $I(2, \mathcal{A})$ from Lemma 3.2 can be obtained via Sylvester forms. Suppose $\mathcal{A}=\left\{\ell_{1}, \ell_{2}, \ldots, \ell_{n}\right\}$ with $n \geq 4$. Using $A_{1,2,4}=\ell_{1} t_{1,2}-\ell_{4} t_{2,4}$ and $A_{1,3,4}=\ell_{1} t_{1,3}-\ell_{4} t_{3,4}$, and the fact that $\ell_{1}, \ell_{4}$ are linearly independent, we get the matrix equation

$$
\left[\begin{array}{l}
A_{1,2,4} \\
A_{1,3,4}
\end{array}\right]=\left[\begin{array}{ll}
t_{1,2} & -t_{2,4} \\
t_{1,3} & -t_{3,4}
\end{array}\right] \cdot\left[\begin{array}{l}
\ell_{1} \\
\ell_{4}
\end{array}\right] .
$$

The determinant of the content matrix is $t_{1,3} t_{2,4}-t_{1,2} t_{3,4}=-Q_{1,2,3,4}^{1}$.

3.3. Comments on Cohen-Macaulayness of $O T(\mathcal{A}, 2)$. In [14] it is proven that $O T(\mathcal{A})$ is (arithmetically) Cohen-Macaulay. This result is also recovered in [10], by the means of Rees algebra, and special fiber related results. With this late approach, in the spirit of [5], there is the hope that one can prove that $O T(2, \mathcal{A})$ is also Cohen-Macaulay, at least for the case when $k=3$ (in three variables). Indeed, $I_{n-2}(\mathcal{A})$ is linearly presented (from Theorem 2.4), and it is generated by the maximal minors of a $(n-2) \times n$ matrix with linear forms entries (see the last paragraphs of the proof of [19, Proposition 2.1]). But the ideals $I \subset A:=\mathbb{K}[x, y, z]$ considered in [5] are perfect ideals, causing for $A / I$ to be Cohen-Macaulay. In our situation, if $k=3$, $R / I_{n-2}(\mathcal{A})$ is Cohen-Macaulay if and only if $\mathcal{A}$ is a rank 3 generic hyperplane arrangement (see Corollary 2.5). In these conditions, by the same coding theory argument we presented in Section 2.3.2, one has that

$$
I_{n-2}(\mathcal{A})=\left\langle x_{1}, x_{2}, x_{3}\right\rangle^{n-2} \subset R=\mathbb{K}\left[x_{1}, x_{2}, x_{3}\right] .
$$

The special fiber of any power $d$ of the maximal ideal $\mathfrak{m}$ of any ring of homogeneous polynomials with coefficients in a field $\mathbb{K}$ is the $d$-th Veronese algebra. By [3, Theorem 5] (citing Gröbner, [11]), it is (arithmetically) Cohen-Macaulay. From this, with [21, Theorem 4.44], we get furthermore, that the Rees algebra of $\mathfrak{m}^{d}$ is also Cohen-Macaulay. Similar argument shows that if $\mathcal{A}$ is any arrangement of rank 2, then the special fiber and the Rees algebra of $I_{n-2}(\mathcal{A})$ are Cohen-Macaulay (see the proof of Claim 3 in Section 2.3.1).

Despite that this approach leads to very special cases, we are still conjecturing that for any $\mathcal{A}$ of any rank $\geq 2, O T(2, \mathcal{A})$ is Cohen-Macaulay.

\section{REFERENCES}

[1] B. Anzis, M. Garrousian and Ş. Tohăneanu, Generalized star configurations and the Tutte polynomial, J. Algebraic Combin. 46 (2017), 165-187.

[2] B. Anzis and Ş. Tohăneanu, Error-correction of linear codes via colon ideals, J. Algebra 443 (2015), $479-493$.

[3] J. Barshay, Determinantal varieties, monomial semigroups, and algebras associated with ideals, Proc. Amer. Math. Soc. 40 (1973), 16-22.

[4] A. Dória, S. Hassanzadeh and A. Simis, A characteristic-free criterion of birationality, Adv. Math. 230 (2012), $390-413$.

[5] A. Dória, Z. Ramos and A. Simis, Linearly presented perfect ideals of codimension 2 in three variables, J. Algebra 512 (2018), 216-251.

[6] D. Eisenbud, The Geometry of Syzygies, Springer, New York 2005.

[7] D. Eisenbud, Commutative Algebra with a View Toward Algebraic Geometry, Springer, New York 1995.

[8] D. Eisenbud and S. Goto, Linear free resolutions and minimal multiplicity, J. Algebra 88 (1984), 89-133.

[9] M. Garrousian and Ş. Tohăneanu, Minimum distance of linear codes and the $\alpha$-invariant, Advances Appl. Math. 71 (2015), 190-207.

[10] M. Garrousian, A. Simis and Ş. Tohăneanu, A blowup algebra for hyperplane arrangements, Algebra and Number Theory (2018), dx.doi.org/10.2140/ant.2018..101.

[11] W. Gröbner, Über Veronesesche Varietäten und deren Projektionen, Arch. Math. 16 (1965), 257-264.

[12] J. Hong, A. Simis and W. V. Vasconcelos, On the homology of two-dimensional elimination, J. Symbolic Comp. 43 (2008), 275-292.

[13] P. Orlik and H. Terao, Commutative algebras for arrangements, Nagoya Math. J. 134 (1994), 65-73.

[14] N. Proudfoot and D. Speyer, A broken circuit ring, Beiträge Algebra Geom. 47 (2006), 161-166. 
[15] H. Schenck, Resonance varieties via blowups of $\mathbb{P}^{2}$ and scrolls, International Mathematics Research Notices 20 (2011), 47564778.

[16] H. Schenck and Ş. Tohăneanu, The Orlik-Terao algebra and 2-formality, Math. Res. Lett. 16 (2009), 171-182.

[17] H. Terao, Algebras generated by reciprocals of linear forms, J. Algebra 250 (2002), 549-558.

[18] Ş. Tohăneanu, On the De Boer-Pellikaan method for computing minimum distance, J. Symbolic Comput. 45 (2010), $965-974$.

[19] Ş. Tohăneanu, A commutative algebraic approach to the fitting problem, Proc. Amer. Math. Soc. 142 (2014), $659-666$.

[20] Ş. Tohăneanu and A. Van Tuyl, Bounding invariants of fat points using a coding theory construction, J. Pure Appl. Algebra 217 (2013), 269-279.

[21] W. Vasconcelos, Integral Closure: Rees Algebras, Multiplicities, Algorithms, Springer-Verlag, Berlin Heidelberg 2005.

[22] R. Villarreal, Monomial Algebras, 2nd edition, CRC Press, Taylor \& Francis Group 2015. 